\title{
A influência dos ácidos graxos trans na disfunção da célula endotelial e o possível efeito terapêutico do exercício sobre o tecido endotelial como forma de prevenção ou regressão da aterosclerose
}

\author{
Influence of trans fatty acids on endothelial cell dysfunction and \\ possible therapeutic effects of physical activity on endothelial tissue for \\ prevention or regression of atherosclerosis
}

Laureane Nunes Masi, Érica Paula Portioli Silva*

\begin{abstract}
Resumo
O endotélio atua ativamente na regulação do tônus vascular, sintetizando e liberando substâncias vasoativas. A inflamação e os fatores de risco cardiovasculares alteram a homeostase vascular, levando à disfunção endotelial e possível formação de placas de ateroma. O aumento das concentrações plasmáticas de ácidos graxos livres pode causar lipotoxicidade vascular, disfunção do endotélio e, finalmente, aterosclerose. Dieta rica em lipídeos contendo ácidos graxos trans tem correlação positiva com a progressão de doenças cardiovasculares. Mudanças no estilo de vida, na adoção de dieta balanceada e atividade física são estratégias para a prevenção de doenças cardiovasculares e a reabilitação de pacientes. Nesta revisão, discutimos a influência benéfica do exercício físico em aspectos importantes da disfunção endotelial causados pelos ácidos graxos trans, incluindo evidências recentes e/ou ainda não exploradas. Discutimos também quais seriam os mecanismos envolvidos no comprometimento funcional da célula endotelial frente ao aumento de ácidos graxos trans na circulação.
\end{abstract} físico.

Palavras-chave: Ácido graxo trans, endotélio vascular, exercício

\section{Introdução}

O endotélio não é um tecido inerte com células simples que formam uma barreira na parte interna dos vasos sanguíneos, mas atua ativamente na regulação do tônus vascular e em sua estrutura ${ }^{1}$. O endotélio vascular é sensível a fatores humorais e hormonais, sintetizando e liberando substâncias vasoativas. A homeostase vascular é mantida pelo balanço entre fatores de contração e dilatação derivados do endotélio. Com a interrupção desse balanço, que ocorre durante a resposta inflamatória e é induzida por fatores de risco cardiovasculares, a vasculatura se torna

\begin{abstract}
The endothelium actively participates in the regulation of vascular tone through the synthesis and release of vasoactive mediators. Inflammation and cardiovascular risk factors affect vascular homeostasis, causing endothelial dysfunction and atheromatous plaque formation. Increased free fatty acid concentrations may result in vascular lipotoxicity, endothelium dysfunction and, ultimately, atherosclerosis. A lipid-rich diet including trans fatty acids has a positive correlation with the progression of cardiovascular diseases. Lifestyle changes, such as eating a well-balanced diet and participating in regular physical activity, have been proposed to prevent cardiovascular diseases and improve rehabilitation. In this review, we discuss the beneficial effects of regular exercise on important aspects of endothelial dysfunction caused by trans fatty acids, including recent and/or yet-to-be-described evidence. The discussion also comprehends the mechanisms involved in endothelial cell dysfunction due to increased trans fatty acid concentrations.
\end{abstract}

Keywords: Trans fatty acids, vascular endothelium, physical activity.

suscetível à formação de placas de ateroma ${ }^{2}$. A disfunção do endotélio refere-se à diminuição na vasodilatação dependente desse ou na liberação de óxido nítrico e também na inibição da expressão ou atividade da enzima óxido nítrico sintase endotelial. As condições mais comuns que predispõem o indivíduo à aterosclerose, tais como hipercolesterolemia, hipertensão, diabetes e o uso de tabaco, estão associadas à disfunção endotelial, induzindo um fenótipo pró-inflamatório e pró-trombótico do endotélio ${ }^{1}$. O aumento nas concentrações plasmáticas de ácidos graxos livres causa redução na atividade da enzima óxido nítrico

\footnotetext{
* Departamento de Fisiologia e Biofísica, Instituto de Ciências Biomédicas, Universidade de São Paulo, (USP), São Paulo, SP.

Não foram declarados conflitos de interesse associados à publicação deste artigo.

Artigo submetido em 10.10.08, aceito em 06.03.09.

J Vasc Bras. 2009;8(2):171-176.

Copyright @ 2009 by Sociedade Brasileira de Angiologia e de Cirurgia Vascular
} 
sintase endotelial e consequente diminuição do óxido nítrico, levando a um aumento na produção de ânion superóxido pelo endotélio ${ }^{3}$. Assim, o aumento do conteúdo de lipídeos plasmáticos é um fator de lipotoxicidade vascular, disfunção do endotélio e aterosclerose ${ }^{4}$.

Atualmente há grande interesse sobre o efeito da dieta rica em ácidos graxos trans e a progressão de doenças cardiovasculares. Em estudos epidemiológicos, foi demonstrado que um aumento de $2 \%$ no consumo de ácidos graxos trans está associado à elevação no risco de doenças coronárias ${ }^{5}$. A associação entre a ingestão de ácidos graxos trans e o risco de doenças cardiovasculares é mais evidente do que aquela entre a ingestão de ácidos graxos saturados e o risco de doenças cardiovasculares. Apesar da eliminação dos ácidos graxos trans da maior parte dos produtos industrializados, ainda não se sabe quais são os mecanismos que afetam a integridade do endotélio vascular.

\section{Disfunção endotelial e ácidos graxos trans: formação de células espumosas?}

Em resposta a estímulos patológicos tais como hipertensão, diabetes e hipercolesterolemia, o funcionamento do endotélio é alterado, levando à disfunção endotelial ${ }^{6,7}$. A disfunção endotelial é caracterizada pela ativação crônica do endotélio, que inclui a produção aumentada de citocinas pró-inflamatórias ${ }^{8}$ e a diminuição da capacidade de vasodilatação dependente de óxido nítrico ${ }^{9}$. Além disso, ocorrem alterações na produção de prostaglandinas e fatores hiperpolarizantes derivados do endotélio, bem como produção aumentada de ânion superóxido (levando à inativação do óxido nítrico) e ativação pró-inflamatória e prótrombótica do endotélio ${ }^{10}$. A homeostase normal de ácidos graxos envolve o balanço entre a formação ou liberação desses pelo tecido adiposo e a sua utilização por tecidos como músculos e fígado. As células acumulam ácidos graxos na forma de triglicerídeos quando a oferta ultrapassa o que é direcionado para anabolismo ou catabolismo. O tecido adiposo estoca grande quantidade de ácidos graxos na forma de triacilgliceróis, enquanto em outros tecidos a capacidade de estoque de lipídeos é limitada. A lipotoxicidade ocorre quando há excesso de lipídeos acumulados, resultando em disfunção celular ou apoptose ${ }^{11}$.

Após o sucesso de hidrogenação de óleos vegetais em 1897, a ingestão de isômeros trans de ácidos graxos insaturados teve aumento significativo na dieta humana ${ }^{12}$. Tais isômeros estão presentes em alimentos industrializa- dos como biscoitos, salgadinhos e margarina. Os ácidos graxos trans constituem uma classe de ácidos graxos insaturados que possuem na sua cadeia uma dupla ligação na configuração trans. Geralmente, esses ácidos graxos ocorrem naturalmente na gordura de ruminantes formada por hidrogenação enzimática de diversos ácidos graxos poliinsaturados (como o ácido linoleico) no rúmen. Também são formados durante o processo industrial de hidrogenação de óleos vegetais. Embora a gordura de ruminantes e os óleos vegetais parcialmente hidrogenados contenham o mesmo isômero de ácido graxo trans, o perfil isomérico é claramente diferente. Na gordura ruminante, o 11 trans-18:1 (11 trans-18:1; ácido vacênico), representa 60-80\% do total $^{13}$, e os óleos vegetais parcialmente hidrogenados têm essencialmente o 9 trans-18:1 (ácido eláidico) ${ }^{14}$. O efeito da ingestão de ácidos graxos trans na saúde humana ainda vem sendo estudado ${ }^{15}$, mas está correlacionado a uma variedade de disfunções, principalmente doenças cardiovasculares. Em vários estudos epidemiológicos foi correlacionada a ingestão elevada de ácidos graxos trans ao aumento de morbidade e mortalidade causadas por doenças cardiovasculares ${ }^{16}$. Em estudo realizado com 730 mulheres, o aumento na ingestão de ácidos graxos trans foi associado ao aumento da expressão de moléculas de adesão intercelular 1 (ICAM-1) e molécula de adesão vascular 1 (VCAM-1), e na concentração de E-selectina no plasma, que são marcadores de disfunção endotelial ${ }^{17}$.

Em comparação à gordura saturada ou cis-insaturada, os ácidos graxos trans elevam o conteúdo de colesterol-LDL (lipoproteína de baixa densidade), reduz o colesterol-HDL (lipoproteína de alta densidade) e aumenta o colesterol total. A proporção reduzida de colesterol-HDL é um fator de risco no desenvolvimento de doenças cardiovasculares ${ }^{18}$. A relação entre a ingestão de ácidos graxos trans e a incidência de doenças cardiovasculares reportada em estudos prospectivos é maior do que poderia ser predito por mudanças nos conteúdos de lipídeos séricos ${ }^{19-21}$, sugerindo que os ácidos graxos trans podem influenciar também outros fatores de risco de doenças cardiovasculares.

O mecanismo básico dos efeitos dos ácidos graxos trans no processo inflamatório e na função endotelial ainda não está bem estabelecido. No entanto, o efeito de outros ácidos graxos são conhecidos na via intracelular, assim como as respostas pela incorporação de ácidos graxos nos fosfolipídeos de membrana, alterando a função de 
receptores específicos ${ }^{22}$ e pela ligação direta e modulação de receptores nucleares regulando a transcrição de genes ${ }^{23}$. Os efeitos de ácidos graxos trans são mediados pelas mesmas vias. Por exemplo, ácidos graxos trans são incorporados na membrana de células endoteliais, monócitos/macrófagos e adipócitos, afetando as vias de sinalização relacionadas à inflamação ${ }^{24}$. A ingestão de ácidos graxos trans altera a resposta inflamatória de células mononucleares em cultura.

Os ácidos graxos trans ativam o sistema inflamatório, elevando os conteúdos de interleucina-6 (IL-6), fator de necrose tumoral- $\alpha$ (TNF- $\alpha$ ), receptores de fator de necrose tumoral e proteína quimioatrativa-1 de monócito $(\mathrm{MCP}-1)^{25}$. Também promovem disfunção vascular, pela redução no fluxo sanguíneo da artéria braquial ${ }^{26}$.

Há mais de 2 décadas, Kinsella et al. ${ }^{27}$ e mais recentemente Kummerow et al. ${ }^{28}$ demonstraram que os ácidos graxos trans influenciam o balanço de prostaglandinas, o que causa trombogênese e inibe a conversão do ácido linoleico em ácido araquidônico, modificando o metabolismo de ácidos graxos e causando mudanças na composição dos ácidos graxos fosfolipídeos na aorta de ratos machos Wistar.

O efeito modificador no índice de massa corporal observado nas relações entre a ingestão de ácidos graxos trans e conteúdos circulantes de interleucina- 6 e proteína C-reativa sugere que os efeitos pró-inflamatórios podem ser parcialmente mediados por respostas do tecido adipo$\mathrm{so}^{29}$. Assim como outros ácidos graxos ${ }^{30}$, os ácidos graxos trans podem se ligar a receptores nucleares de adipócitos e de outros tecidos, incluindo o receptor ativado por proliferador de peroxissomas (PPAR-gama), receptor $\mathrm{x}$ do fígado, e proteína ligante do elemento regulatório de esterol-1, regulando genes que afetam fatores de risco cardiovasculares por ação de lipídeos ou não-lipídeos. Em estudos realizados em animais, a ingestão de ácidos graxos trans alterou a expressão de genes em adipócitos tais como PPAR-gama, resistina e lipase de lipoproteína ${ }^{31}$. Os ácidos graxos trans também influenciam o metabolismo de outros ácidos graxos em adipócitos humanos ${ }^{32}$.

Nesses estudos ficou demonstrado que os ácidos graxos trans estão ligados ao desenvolvimento de doenças cardiovasculares, provavelmente por ativar resposta próinflamatória vascular ${ }^{33}$. Contudo, a forma e a extensão pela qual os ácidos graxos trans afetam a vasculatura ainda são desconhecidas.

\section{Efeito do exercício físico na disfunção endotelial}

A associação entre fatores de risco coronários e doenças cardiovasculares com a disfunção endotelial indica que a intervenção na redução dos fatores de risco e a melhora da função endotelial devem reduzir a isquemia coronária e síndromes coronárias instáveis. As intervenções incluem terapia com estatina, estrogênio, vitaminas $\mathrm{E}$ e $\mathrm{C}$, inibidores da enzima conversora de angiotensina e exercício físico.

O exercício físico reduz os riscos cardiovasculares. Embora a relação entre exercício aeróbico e vasodilatação dependente do endotélio apresenta correlação positiva, poucos estudos foram realizados nessa área. Além disso, os estudos utilizam diferentes tipos de exercício e populações diversificadas (normal, hipertensivo, pacientes coronários). Assim, cada situação precisa ser considerada individualmente, e os resultados não podem ser generalizados. Hambrecht et al. ${ }^{34}$ demonstraram que 4 semanas de treinamento físico vigoroso melhora a disfunção endotelial em pacientes com sintomas de doenças cardiovasculares. A vasoconstrição coronária em resposta à infusão de acetilcolina foi atenuada após o treinamento físico de 4 semanas, indicando que o exercício causou efeitos benéficos no endotélio de artérias do epicárdio coronário. Também foi demonstrado que o exercício físico está associado ao aumento de mediadores agonistas da velocidade do fluxo sanguíneo. A regressão final em lesões coronárias e recrutamento do fluxo colateral, que seria considerada improvável num período de 4 semanas, foram atenuadas. $\mathrm{Kruk}^{35}$ mostrou o efeito positivo do treinamento físico e a melhora na perfusão miocárdica, enfatizando o efeito potencial da terapia de treinamento físico para pacientes com doenças cardiovasculares $^{35}$.

Em outro estudo, foi verificado que o exercício aeróbico regular pode prevenir a perda de vasodilatação dependente do endotélio, além de restabelecer valores prévios em homens sedentários de meia-idade e idosos ${ }^{36}$. Homens submetidos a treinamento aeróbico não tiveram vasodilatação dependente do endotélio preservada quando comparados a sedentários ${ }^{37}$.

Clarkson et al. ${ }^{38}$ encontraram resultados positivos em 10 semanas de treinamento com programa de exercícios 
aeróbicos e anaeróbicos em soldados saudáveis. Gokce et al. ${ }^{39}$ também encontraram aumento significativo na vasodilatação dependente do endotélio da artéria tibial posterior com 10 semanas de exercício aeróbico moderado, com resultados positivos principalmente nas pernas.

Higashi et al. ${ }^{40}$ analisaram indivíduos hipertensos submetidos a um programa de exercício aeróbico por 12 semanas (30 minutos, 5 a 7 vezes por semana) e encontraram aumento significativo na vasodilatação dependente do endotélio em resposta à acetilcolina. Indivíduos com infarto agudo do miocárdio submetidos a 3 meses de treinamento físico aeróbico (ciclo ergométrico) com intensidade de $75 \%$ do pico de exercício máximo do coração também apresentaram aumento na vasodilatação dependente do endotélio. Entretanto, com 1 mês sem treinamento, os benefícios desapareceram. Tais resultados são sugestivos de que indivíduos com históricos de eventos cardíacos podem obter melhora na função endotelial ${ }^{41}$.

Goto $^{42}$ analisou o efeito de diferentes intensidades de exercício aeróbico nas artérias e verificou que a intensidade moderada ( $50 \% \mathrm{VO}_{2}$ máxima) foi o único capaz de melhorar a vasodilatação dependente do endotélio. Exercício de baixa ( $25 \% \mathrm{VO}_{2}$ máxima) e alta $\left(75 \% \mathrm{VO}_{2}\right.$ máxima $)$ intensidade não promoveram qualquer beneficio na função endotelial. O exercício de alta intensidade levou a um aumento no estresse oxidativo, aspecto prejudicial para a parede arterial. Por essa razão, o exercício de intensidade moderada parece ser o mais apropriado para indivíduos sedentários que procuram benefícios cardiovasculares através de exercício aeróbico regular.

Em estudo realizado por Umpierre \& $\operatorname{Stein}^{43}$, com homens sedentários saudáveis idosos e de meia-idade randomizados para treinamento físico de resistência por 13 semanas, foi mostrado aumento significativo no fluxo sanguíneo e na condutância vascular na artéria femoral. Em ratos knockout $\mathrm{db} / \mathrm{db}$ diabéticos tipo 2 e normoglicêmicos submetidos a exercício de intensidade moderada, o treinamento físico reverteu a disfunção endotelial diabética vascular independentemente de regredir o peso corporal ou o estado hiperglicêmico ${ }^{44}$. Embora diversos pesquisadores tenham demonstrado que o exercício aeróbico regular melhora a vasodilatação dependente do endotélio, não se sabe ainda o quanto o treinamento responde por essas mudanças ${ }^{45}$.
$\mathrm{O}$ aumento na capacidade do exercício aeróbico $\left(\mathrm{VO}_{2}\right.$ máxima) foi associado ao aumento no diâmetro arterial $(\mathrm{r}=0,66 \mathrm{p}<0,002)$ como resposta à hiperemia em homens idosos treinados $(68,5 \pm 2,3$ anos) comparados a idosos sedentários $(64,7 \pm 1,4 \text { anos })^{46}$. Essa associação resultou de maior capacidade de vasodilatação dependente do endotélio nos indivíduos que treinaram ao menos três vezes por semana por no mínimo 1 hora por dia. Tem sido demonstrado que o treinamento físico em pacientes com doença arterial coronária estável promove redução de $49 \%$ na vasoconstrição induzida por angiotensina II. Tal adaptação foi acompanhada também por menor expressão do receptor AT1 e aumento da expressão do receptor AT2 $2^{47}$.

A Organização Mundial da Saúde recomenda atividade física aeróbica de intensidade moderada como promoção da saúde, mas é necessário compreender o quanto o exercício de intensidade moderada causa efeito anti-inflamatório. Markovitch et al. ${ }^{48}$ demonstraram que o exercício agudo de intensidade moderada $\left(50 \% \mathrm{VO}_{2}\right.$ máxima) não causa mudanças significativas na circulação de neutrófilos, monócitos ou na concentração sérica de citocinas como interleucina-6, interleucina-10 e proteína $\mathrm{C}$ reativa, depois de 7 dias consecutivos de exercício em homens adultos saudáveis ( $54 \pm 4 \mathrm{anos})$. Esses resultados são indicativos de que o exercício agudo em intensidade moderada pode não causar os mesmos efeitos benéficos encontrados em estudos com programa de treinamento de exercícios crônicos de intensidade moderada conforme observado nos estudos citados e recomendados pela Organização Mundial da Saúde.

Como visto, o exercício aeróbico melhora e restaura a vasodilatação dependente do endotélio, independente do tipo de esforço e da intensidade.

\section{Considerações finais}

Nessa breve revisão, observamos que há um efeito direto do ácido graxo trans no estado de ativação e na função de células endoteliais, apesar de ainda não estar claro os mecanismos pelos quais tais isômeros causam disfunção endotelial. O exercício físico tem sido usado como intervenção terapêutica, e há correlação positiva entre treinamento e aumento na vasodilatação dependente do endotélio. Os mecanismos pelos quais o exercício físico atua nessa melhora da vasodilatação dependente do endotélio são ainda desconhecidos. Mais estudos nesse assunto 
devem ser realizados para determinar o mecanismo de ação dos ácidos graxos trans que leva a disfunção da célula endotelial e o possível efeito terapêutico da prática da atividade física sobre o tecido endotelial como forma de prevenção ou regressão da aterosclerose.

\section{Agradecimentos}

Agradecemos ao CNPq, CAPES e FAPESP pelo apoio financeiro. Alguns trechos do texto foram modificados por sugestão do professor Rui Curi.

\section{Referências}

1. Landmesser U, Horning B, Drexler H. Endothelial function: a critical determinant in atherosclerosis? Circulation. 2004;109:II27-33.

2. Szmitko PE, Wang CH, Weisel RD, de Almeida JR, Anderson TJ, Verma S. New markers of inflammation and endothelial cell activation. Part I. Circulation. 2003;108:1917-23.

3. Steinberg D. A critical look at the evidence for the oxidation of LDL in atherogeneses. Atherosclerosis. 1997;131 Suppl:S5-7.

4. Mattern HM, Hardin CD. Vascular metabolic dysfunction and lipotoxicity. Physiol Res. 2007;56:149-58.

5. Stender S, Dyerberg J. Influence of trans fatty acids on health. Ann Nutr Metab. 2004;48:61-6.

6. Jorge PA, Osaki MP, de Almeida E, Cledídio Neto L, Metze $\mathrm{K}$. Effects of vitamin $\mathrm{E}$ on endothelium-dependent coronary flow in hypercholesterolemic dogs. Atherosclerosis. 1996;126:43-51.

7. Grundy SM, Denke MA. Dietary influences on serum lipids and lipoproteins. J Lipid Res. 1990;31:1149-72.

8. Shaw DI, Hall WL, Jeffs NR, Williams CM. Comparative effects of fatty acids on endothelial gene expression. Eur $\mathrm{J}$ Nutr. 2007;46:321-8.

9. Bartus M, Lomnicka M, Lorkowska B, et al. Hypertriglyceridemia but not hypercholesterolemia induces endothelial dysfunction in the rat. Pharmacol Rep. 2005;57 Suppl:12737.

10. Bonetti PO, Lerman LO, Lerman A. Endothelial dysfunction: a marker of atherosclerotic risk. Arterioscler Thromb Vasc Biol. 2003;23:168-75.

11. Schaffer JE. Lipotoxicity: when tissues overeat. Curr Opin Lipidol. 2003;14:281-7.

12. Emken EA. Nutrition and biochemistry of trans and positional fatty acid isomers in hydrogenated oils. Annu Rev Nutr. 1984;4:339-76.

13. Kraft J, Collomb M, Mockel P, Sieber R, Jahreis G. Differences in CLA isomer distribution of cow's milk lipids. Lipids. 2003;38:657-64.

14. Aro A, Pietinen P, Valsta LM, et al. Effects of reduced-fat diets with different fatty acid compositions on serum lipoprotein lipids and apolipoproteins. Public Health Nutr. 1998;1:109-16.

15. Dyerberg J, Eskesen DC, Andersen PW, et al. Eur J Clin Nutr. 2004;58:1062-70.
16. Woodside JV, McKinley MC, Young IS. Saturated and trans fatty acids and coronary heart disease. Curr Ather Rep. 2008; 10:460-6.

17. Lopez-Garcia E, Schulze MB, Meigs JB, et al. Consumption of trans fatty acids is related to plasma biomarkers of inflammation and endothelial dysfunction. J Nutr. 2005;135:562-6.

18. Stampfer MJ, Sacks FM, Salvini S, Willett WC, Hennekens $\mathrm{CH}$. A prospective study of cholesterol, apolipoproteins, and the risk of myocardial infarction. $\mathrm{N}$ Engl $\mathrm{J}$ Med. 1991;325:373-81.

19. Ascherio A, Katan MB, Zock PL, Stampfer MJ, Willett WC. trans fatty acids and coronary heart disease. N Engl J Med. 1999;340:1994-8.

20. Katan MB. Regulation of trans fats: the gap, the Polder, and McDonald's French fries. Atheroscler Suppl. 2006;7:63-6.

21. Mensink RP, Zock PL, Kester AD, Katan MB. Effects of dietary fatty acids and carbohydrates on the ratio of serum total to HDL cholesterol and on serum lipids and apolipoproteins: a meta-analysis of 60 controlled trials. Am J Clin Nutr. 2003;77:1146-55.

22. Kuhn N, Willet A. [Nursing in Europe-Luxemborg: developing and utilizing new nursing fields]. Pflege Z. 2006;59:1825.

23. Hennig B, Alvarado A, Ramasamy S, Boissonneault GA, Decker E, Means WJ. Fatty acid induced disruption of endothelial barrier function in culture. Biochem Arch. 1990;6:409-17.

24. Hennig B, Toborek M, McClain CJ, Diana JN. Nutritional implications in vascular endothelial cell metabolism. J Am Coll Nutr. 1996;15:345-58.

25. Mozaffarian D, Rimm EB, King IB, Lawler RL, McDonald GB, Levy WC. trans fatty acids and systemic inflammation in heart failure. Am J Clin Nutr. 2004;80:1521-5.

26. de Roos NM, Bots ML, Katan MB. Replacement of dietary saturated fatty acids by trans fatty acids lowers serum HDL cholesterol and impairs endothelial function in healthy men and women. Arterioscler Thromb Vasc Biol. 2001;21:12337.

27. Kinsella JE, Bruckner G, Mai J, Shimp J. Metabolism of trans fatty acids with emphasis on the effects of trans, transoctadecadienoate on lipid composition, essential fatty acid, and prostaglandins: an overview. Am J Clin Nutr. 1981;34:2307-18.

28. Kummerow FA, Zhou Q, Mahfouz MM, Smiricky MR, Grieshop CM, Schaeffer DJ. trans fatty acids in hydrogenated fat inhibited the synthesis of the polyunsaturated fatty acids in the phospholipid of arterial cells. Life Sci. 2004;74:2707-23.

29. Toborek M, Barger SW, Mattson MP, Barve S, McClain CJ, Hennig B. Linoleic acid and TNF-a cross-amplify oxidative injury and dysfunction of endothelial cells. J Lipid Res. 1996;37:123-35.

30. Hennig B, Shasby DM, Spector AA. Exposure to fatty acid increases human low density lipoprotein transfer across cultured endothelial monolayers. Circul Res. 1985;57:776-80.

31. Hennig B, Meerarani P, Ramadass P, Watkins BA, Toborek M. Fatty acid-mediated activation of vascular endothelial cells. Metabolism. 2000;49:1006-13. 
32. Hennig B, Shasby DM, Fulton AB, Spector AA. Exposure to free fatty acid increases the transfer of albumin across cultured endothelial monolayers. Arterosclerosis. 1984;4:48997.

33. Harvey KA, Arnold T, Rasool T, Antalis C, Miller SJ, Siddiqui RA. trans-fatty acids induce pro-inflammatory responses and endothelial cell dysfunction. $\mathrm{Br} \mathrm{J}$ Nutr. 2008;99:723-31.

34. Hambrecht R, Hilbrich L, Erbs S, et al. J Am Coll Cardiol. 2000;35:706-13.

35. Kruk J. Asian Pac J Cancer Prev. 2007;8:325-38.

36. De Souza CA, Shapiro LF, Clevenger CM, et al. Regular aerobic exercise prevents and restores age-related declines in endothelium-dependent vasodilation in healthy men. Circulation. 2000;102:1351-7.

37. Galleta F, Franzoni F, Virdis A, et al. Endotheliumdependent vasodilation and carotid artery wall remodeling wall in athletes and sedentary subjects. Atherosclerosis. 2006;186:184-92.

38. Clarkson P, Montgomery HE, Mullen MJ, et al. Exercise training enhances endothelium function in young men. J Am Coll Cardiol. 1999;33:1379-85.

39. Gokce N, Vita JA, Bader DS, et al. Effect of exercise on upper and lower extremity endothelium function in patients with coronary artery disease. Am J Cardiol. 2002;90:124-7.

40. Higashi Y, Sasaki S, Kurisu S, et al. Regular aerobic exercise augments endothelium-dependent vascular relaxation in normotensive as well as hypertensive subjects: role of endothelium-derived nitric oxide. Circulation. 1999;100:1194202.

41. Vona M, Rossi A, Capodaglio P, et al. Impact of physical training and detraining on endothelium-dependent vasodila- tion in patients with recent acute myocardial infarction. Am Heart J. 2004;147:1039-46.

42. Goto Y. [Exercise therapy for chronic heart failure]. Nippon Rinsho. 2003;61:851-6.

43. Umpierre D, Stein R. Hemodynamic and vascular effects of resistance training: implication for cardiovascular disease. Arq Bras Cardiol. 2007;89:256-62.

44. Moien-Afshari F, Ghosh S, Elmi S, et al. Exercise restores endothelial function independently of weight loss or hyperglycemic status in db/db mice. Diabetologia. 2008;51:132737.

45. Sasaki JE, Santos MG. The role of aerobic exercise on endothelial function and on cardiovascular risk factors. Arq Bras Cardiol. 2006;87:e226-31.

46. Rinder MR, Spina RJ, Ehsani AA. Enhanced endotheliumdependent vasodilation in older endurance-trained men. J Applied Physiol. 2000;88:761-6.

47. Adams J, Cline MJ, Hubbard M, McCullough T, Hartman J. A new paradigm for post-cardiac event resistance exercise guidelines. Am J Cardiol. 2006;97:281-6.

48. Markovitch D, Tyrrell RM, Thompson D. Acute moderate-intesity exercise in middle-aged men has neither an antinor proinflammatory effect. J Appl Physiol. 2008;105:260-5.

Correspondência:

Laureane Nunes Masi

Avenida Prof. Lineu Prestes, 1524, sala 105

Instituto de Ciências Biomédicas I, Cidade Universitária, Butantã

CEP 05508-900 - São Paulo, SP

Tel.: (11) 3091.7245

Fax: (11) 3901.7285

E-mail: laure_masi@hotmail.com 